\title{
FRACASO UNIVERSITARIO: ¿ILUSIÓN O REALIDAD?
}

\author{
COLLEGE FAILURE: ILLUSION OR REALITY?
}

\author{
Ángel Luis Pompa*, Noemí Ponz*, Vanessa Rama*, José Antonio Ríos** \\ Universidad Complutense
}

\section{RESUMEN}

Son numerosos los estudios que hacen referencia al fracaso escolar, pero pocos los que se centran en la etapa universitaria. Diversos factores condicionan la elección de carrera, la valoración del profesorado, la atribución (externa o interna) de los resultados o las consecuencias del suspenso. Cada uno de ellos se manifiesta en mayor o menor grado en diferentes carreras universitarias.

\begin{abstract}
They are enormous the studies which make reference to the school failure but few of them are adjusted to the university aged. Several key factors put into working order the choice of the career, the evaluation of the teaching staff, the attribution (eexternal or internal) of the results of the consecuencies of the fail. Each of them are revealed with more or less importance in differents university careers.
\end{abstract}

\section{Introducción}

Las características innatas del individuo junto con las adquiridas por contacto con el medio que le rodea marcan las relaciones entre la persona y la sociedad. Su conducta está determinada por el entorno físico y social y la respuesta específica en cada situación.

En concreto, el rendimiento académico del alumno es resultado de la interacción entre el contexto educativo (institución, profesores, compañeros, ...), la capacidad intelectual del sujeto, su motivación, personalidad y ambiente familiar y social en el que se desenvuelve. De-

* Alumno o alumna de la asignatura de «Orientación Educativa» (II Ciclo) en la Facultad de Psicología de la UCM durante el curso 1999-2000, bajo la docencia del Prof. José A. Ríos. Actualmente Licenciados en Psicología, especialidad de Psicología de la Educación.

** Profesor Titular de la asignatura de «Orientación Educativa» en dicha Facultad y Fundador-Director del Servicio de Orientación Educativa en la misma Facultad desde su fundación (1988) hasta 2001, fecha de su jubilación. Este Servicio ha sido el primero constituido en la Universidad Española con ininterrumpida vida y actividad entre las fechas citadas. 
pendiendo de dicha interacción se pueden producir dos consecuencias opuestas dentro del rendimiento: éxito o fracaso.

En cuanto al fracaso es difícil encontrar una definición completa y unánime debido a que las diferentes descripciones se centran en aspectos tan dispares como la insuficiencia de capacidad intelectual, la falta de motivación, la repetición de curso, el rendimiento insatisfactorio, etc.

McClelland y Atkinson (1964) definen el fracaso como «la situación de inhibición afectiva ante el estudio después de múltiples experiencias de fracaso de alumnos poco motivados para el logro».

Wall (1970) lo define como «una discrepancia entre lo que se podría rendir en circunstancias relativamente favorables y lo que de hecho se rinde».

Ríos González (1973) lo entiende como «la situación en la que el sujeto no consigue alcanzar las metas normales para el grado de inteligencia que posee, de tal modo que toda su personalidad queda comprometida y alterada, repercutiendo en su rendimiento global como persona y en su adaptación sana y eficaz al tipo de vida que le rodea».

Avanzini (1979) entiende que «el fracaso no es un estado puramente objetivo que corresponde a unos datos rigurosos y universales, sino a una situación creada por las malas notas, la repetición del curso, el retraso o el suspenso; no se vive necesariamente, ni se siente, como una situación penosa si el individuo y su familia, con razón o no, son indiferentes al rendimiento escolar»».

Rubio Jerónimo (1989), hace referencia al fracaso escolar como un revelador de desaprovechamientos, malogros, aspiraciones insatisfechas y resultados académicos adversos que tiene como consecuencia retrasos indebidos o estancamientos forzados en un contexto donde se reclama un mínimo aprovechamiento (por parte tanto del sistema educativo como del entorno familiar y la sociedad en general) capaz de promocionarle de un curso al siguiente y de un ciclo del sistema educativo a otro superior; logrando una autorrealización personal como individuo y una promoción social y personal.

Portellano (1989) lo considera como «el desfase negativo entre la capacidad intelectual del alumno y su rendimiento escolar».

González Tirados (1989) precisa que el fracaso es la no consecución por parte del alumno de las metas formativas que se fijan en un determinado nivel educativo.

Tierno (1997) diferencia entre fracaso escolar y suspenso y afirma que las malas notas no siempre son signo de fracaso escolar. Además, para hablar de fracaso escolar hay que tener en cuenta la relación entre: capacidad intelectual y rendimiento personal, nivel de rendimiento exigible oficialmente y rendimiento personal, capacidades individuales y rendimiento personal y nivel personal de aspiraciones.

Tras haber realizado un exhaustivo análisis de diversas publicaciones hemos observado que existe consenso a la hora de señalar cuáles son los factores que influyen en el rendimiento, en especial el universitario. Latiesa (1992) enumera los siguientes, situándolos dentro de diferentes aproximaciones teóricas:

1. El origen social: dentro de este aspecto podemos diferenciar el modelo de respuesta, que considera los orígenes sociales en sí y el modelo de situación que hace referencia a la atmósfera universitaria. 
La familia es el medio «donde se aprenden roles, modelos y patrones de comportamiento que inciden en los comportamientos académicos y en el aprendizaje».

2. Características personales, familiares y escolares: como características personales destacan la edad (los jóvenes tienen más éxito), el sexo (que no influye en el rendimiento académico pero existe una ligera tendencia en la mujer a tener más éxito) y la actividad laboral (abandonan más los alumnos que trabajan). Algunas características familiares como la profesión o los estudios de los padres no influyen en el rendimiento. Las características escolares son las más importantes a la hora de predecir el rendimiento (calificaciones, tipo de escolaridad, tipo de acceso y opción cursada en secundaria).

3. Características del medio universitario: «Variables como tamaño o número de alumnos, relación profesor-alumno, número de libros de la biblioteca, y el clima o medio institucional influyen en el rendimiento».

4. Características psicológicas: la inteligencia está relacionada con el rendimiento pero esta relación es menor cuanto mayor es el nivel de estudios. Aptitud numérica, razonamiento abstracto y tests lógico y verbal, todos ellos factores cognitivos, no predicen el rendimiento universitario. La motivación está más relacionada con el rendimiento académico que con la personalidad del estudiante. Aunque existen conclusiones contradictorias, el éxito puede ser explicado por la satisfacción del alumno con los estudios cursados. El tiempo de estudio y su organización configuran estilos cognitivos de aprendizaje diferentes, correlacionando con la motivación y el compromiso pero poco con el rendimiento.

5. Procesos de acceso a la educación superior: «La oferta y la demanda de plazas condicionan el tipo de alumnado [...] en cada disciplina y consecuentemente, el rendimiento académico».

6. Mercado de trabajo: el esfuerzo y el compromiso por los estudios es mayor en las carreras con posibilidades de empleo.

Rendimiento, fracaso y deserción son términos asociados, ya que el rendimiento se puede considerar desde dos dimensiones: el éxito y el fracaso. Dentro de este último una de las posibles consecuencias es la deserción, cuyos factores condicionantes se exponen a continuación (M. Latiesa, 1992):

1. Oferta de plazas: este factor tiene dos vertientes. La primera, institucional: el número de plazas ofertadas depende de políticas educativas para las que el centro posee casi total libertad en determinar o no numerus clausus. La segunda, individual: se pueden dar, a su vez, dos categorías; los alumnos cursan la opción solicitada o, por el contrario, al ser rechazados en ciertas carreras, cursan cualquier otra opción.

2. Demanda de plazas: este es un proceso previo que determina las inscripciones por carreras. Así, será la primera fuente que distinga a los alumnos que acceden a cada una de ellas. La demanda de plazas viene determinada por los condicionamientos de la elección (la elección de carrera está influida por las limitaciones de la oferta y por las características personales, familiares y escolares del alumno), por los motivos de elección de carrera (tradición familiar, buenas notas en las asignaturas relacionadas con bachiller, carrera fácil, independencia económica futura) y por los intereses, las expectativas y aspiraciones en la elección de carrera. 
3. Características del alumnado: pueden distinguirse tres tipos de características: personales (edad, sexo, actividad laboral y lugar de residencia), familiares (estudios de los padres y profesión de ambos) y escolares (opción cursada en bachiller, tipo de centro, antecedentes académicos y estudios universitarios previos).

4. Otras características del medio universitario y desarrollo de la escolaridad: son las variables que muestran el grado de relación de los alumnos con los estudios (asistencia a clase, horas dedicadas a estudiar, búsqueda de trabajo, satisfacción con los estudios).

5. Dificultad de los estudios: esta no es una variable contrastada con datos empíricos, pero es importante distinguir entre la dificultad subjetiva (anticipación de los alumnos acerca de los estudios que van a cursar y fracaso debido a las características del alumno en concreto) y objetiva (atribuible al centro).

6. Inserción profesional: en general a la hora de elegir carrera se tienen en cuenta diferentes aspectos (por orden de prioridad): posibilidades de encontrar empleo, prestigio e ingresos que se pueden obtener en el futuro. El hecho de que existan posibilidades de empleo inmediato genera alto grado de compromiso con el estudio en la carrera.

Como conclusión, es importante señalar que el estudio de la deserción universitaria debe llevarse a cabo de forma aislada para cada tipo de estudios, ya que la Universidad es una entidad heterogénea. En el estudio de la realidad social universitaria es necesario un análisis cuantitativo y cualitativo ya que ambos se requieren y complementan. Existen diferentes variables que influyen en la deserción universitaria y por ello, aunque sería necesario un modelo único para su interpretación, debe asumirse una pluralidad metodológica a causa de una falta de consenso entre los diferentes modelos presentados por los autores.

\section{Fracaso universitario: un estudio de 1995}

Según los estudios realizados en este período (Estudio del Fracaso Escolar en la U.C.M. Servicio y asignatura de «Orientación Educativa». Facultad de Psicología, bajo la dirección de José Antonio Ríos González y coordinando esta investigación Luis Cerrón, actualmente Profesor en la Escuela Universitaria Cardenal Cisneros de la Universidad de Alcalá de Henares) se encuentran diferencias importantes entre los datos obtenidos con alumnos pertenecientes a distintas carreras (Farmacia, Veterinaria, Químicas, Ciencias de la Educación y Psicología, todas ellas Facultades de la U.C.M.). La muestra utilizada fue de 453 sujetos distribuidos de la siguiente forma: Farmacia 87, Veterinaria 70, Químicas 78, Ciencias de la Educación 65 y Psicología 153. Cada uno de los sujetos respondió a un cuestionario formado por un total de 20 preguntas entre las cuales se incluían algunas con respuesta abiertas y otras con diferentes alternativas.

En cuanto a los motivos de la elección de carrera podemos diferenciar entre endógenos (entendiendo por motivación endógena aquella que es originada dentro del propio organismo, no debida a una causa exterior) y exógenos (se entiende por motivación exógena aquella procedente del exterior, debida a una causa exterior, no generada por el mismo organismo), siendo los primeros los de mayor importancia para los alumnos que cursan dichas carreras (véase gráfica 1). Veterinaria (92\%) y Psicología (89\%) son las dos carreras donde 
los factores endógenos cobran mayor relevancia, aunque en el resto de carreras las puntuaciones también son elevadas. La vocación profesional (inclinación o deseo permanente y vehemente de la satisfacción a una aptitud mediante su ejercicio en el trabajo [...]. El concepto de vocación incluye tres notas: aptitud, capacitación e interés) es el factor endógeno más destacado aunque las puntuaciones no son tan elevadas como cabría esperar, siendo especialmente significativo en Ciencias de la Educación donde sólo un $28 \%$ elige la carrera por este motivo. Eligen sus estudios teniendo en cuenta el futuro profesional (entendiendo por este, el tener un puesto de trabajo el día de mañana con un buen prestigio, que esté bien reconocido por la sociedad y que sea bien remunerado) un porcentaje considerablemente menor de alumnos siendo más bajo en Psicología (24\%) y en Ciencias de la Educación (26\%) (gráfica 2).

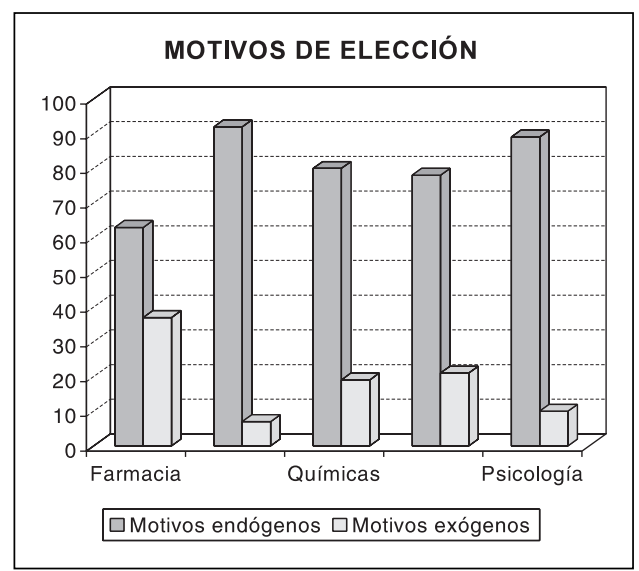

GRÁFICA 1.

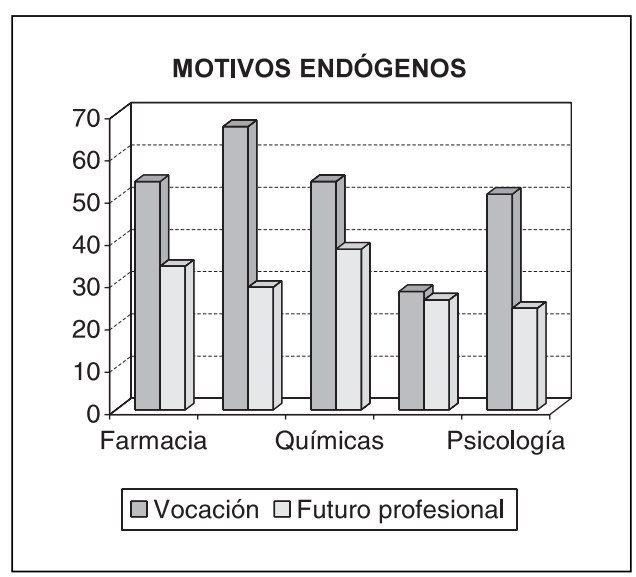

GRÁFICA 2.

Las expectativas del alumno al realizar la carrera elegida se centran en dos aspectos fundamentales: salida profesional y formación. En todas las carreras destaca el primero frente al segundo excepto en Psicología donde están muy igualados (48\% frente a 47\%).

Ejercer tras terminar los estudios superiores es la aspiración prioritaria de los estudiantes (en torno al 55\% en todas ellas). Esta puntuación es bastante alta si la comparamos con el resto de opciones que pueden llevarse a cabo (opositar, cursos de postgrado, estudiar otra carrera, ...).

La proporción de sujetos que no ha suspendido nunca ninguna asignatura en la carrera es muy baja en todas, excepto en Cc. de la Educación, donde el tanto por ciento es muy superior $(38 \%)$. Si consideramos el hecho de haber suspendido un curso completo, el porcentaje disminuye considerablemente dándose como máximo un 6\% en Cc. de la Educación y un $4 \%$ en Psicología. Un suspenso es considerado como un estímulo para estudiar más y superarse en todas las disciplinas excepto en Químicas, donde produce desánimo generalizado en la actividad académica. En el fracaso académico en Farmacia, Químicas y Psicología se realiza una atribución interna (poco tiempo dedicado al estudio), mientras que en Veterinaria y Cc. de la Educación se realiza una atribución externa (malos profesores). 
En general, todos los alumnos tienen una autoimagen positiva en lo que a estudio se refiere. Estos consideran que las características del buen estudiante por orden de importancia son la comprensión lectora, la asimilación de lo estudiado, estar al día, ampliar información, asistir a clase, memorización y simplemente aprobar. Esta jerarquía no se cumple en Cc. de la Educación porque dan más importancia a la asistencia que a la comprensión lectora, que aparece en segundo lugar seguida de ampliar información, asimilar lo estudiado, estar al día y memorización junto con simplemente aprobar en último lugar. La autopercepción de fracaso tiene un porcentaje superior en Veterinaria (15,4\%), seguida de Farmacia $(9,3 \%)$ y Psicología (7,8\%), siendo muy baja en Químicas $(3,8 \%)$ y Cc. de la Educación $(1,5 \%)$.

En cuanto al tiempo dedicado al estudio, los alumnos de Químicas son los que estudian con mayor antelación (un mes antes para asignaturas de dificultad alta y media y 15 días antes en asignaturas consideradas fáciles) frente a los de Cc. de la Educación que comienzan 15 días antes en asignaturas de dificultad alta y media y una semana antes en las de baja dificultad. En Farmacia, Veterinaria y Psicología el tiempo empleado es de un mes para las de elevada dificultad, 15 días para las de dificultad moderada y una semana para las de baja dificultad.

Las disciplinas en las que se producen mayores problemas con el personal docente son Veterinaria (29,2\%) junto con Cc. de la Educación (27,7\%). A continuación se situarían Químicas (23,1\%), Psicología (22,5\%) y Farmacia (20,7\%).

Las características que se atribuyen al buen profesor son: que posea conocimientos y los sepa transmitir (en la gráfica Didáctico), que sea exigente, que apruebe por ir a clase (en la gráfica Asistencia), que sea directivo, que sea crítico-razonable, que fomente la participación, que sea entusiasta y tenga motivación para enseñar, y que permita libertad así como que deje hacer. Estas características se distribuyen de manera desigual en las diferentes carreras (Gráfica 3).

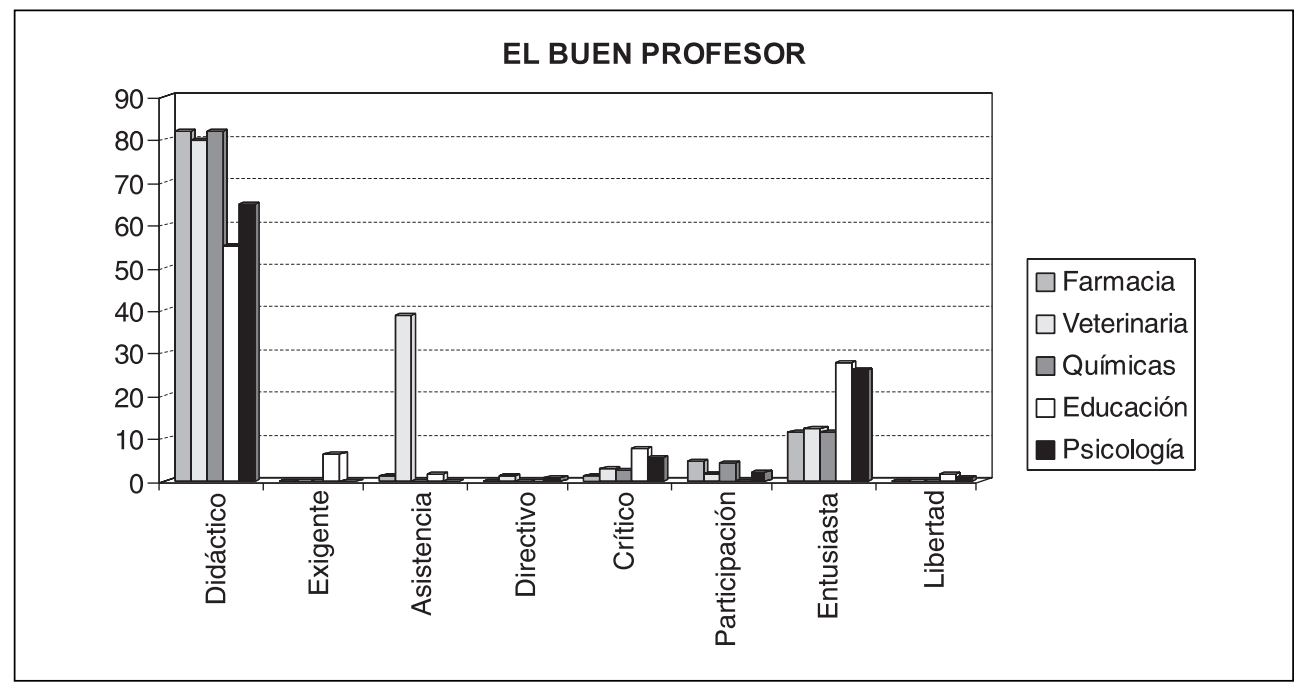

GRÁFICA 3. 
La última cuestión a tener en cuenta es si los alumnos han recibido o no, orientación a lo largo de su vida académica. Aproximadamente la mitad de los alumnos han recibido orientación antes de acceder a sus estudios superiores, destacando el alto porcentaje de los que acceden a Veterinaria que llegan a alcanzar un $60 \%$ frente a los que dirigiéndose a Ciencias de la Educación, y paradójicamente, sólo reciben orientación previa un $43 \%$. Considerando el tanto por ciento de alumnos que no la han recibido, más del $80 \%$ señala que hubiera deseado recibirla excepto en Veterinaria, donde sólo la reclama un 62\%. Entre los que recibieron orientación, alrededor de la mitad fueron guiados en la elección de su carrera, aunque en Cc de la Educación y Psicología esta cifra se sitúa por debajo de un tercio.

Si nos fijamos en los porcentajes de alumnos que están cursando la carrera deseada, Veterinaria destaca en primer lugar con un 90,8\%, seguida de Psicología (84,6\%), Químicas $(79,5 \%)$ y Cc de la Educación (70,8\%). Sin embargo, este porcentaje es mucho menor en Farmacia donde tan sólo llega al 58\%.

Una vez que los alumnos se han incorporado a la Universidad, siguen reclamando un Servicio de Orientación para Universitarios incluso, en mayor medida que en los estudios preuniversitarios. Así, por ejemplo, en Veterinaria esta diferencia es del 18\% aunque sigue siendo la carrera en la que menos orientación se solicita.

\section{Fracaso universitario: Un estudio del 2000}

En el estudio realizado durante el curso 1999-2000, (Estudio de Fracaso Escolar en la U.C.M. Servicio de Orientación Educativa. Facultad de Psicología, fundado y dirigido desde 1988 por el profesor José Antonio Ríos González) en las facultades de Económicas y Psicología de la U.C.M., se trabajó con una muestra total de 422 sujetos, de los cuales 148 pertenecían a Económicas y 274 a Psicología. El cuestionario utilizado fue el mismo que en la investigación del año 1995. Los resultados obtenidos se exponen a continuación:

Tanto los alumnos de Psicología como los de Económicas, eligen su carrera basándose en motivos endógenos (con un porcentaje del 81\% en ambos casos) (Gráfica 4). Dentro de estos, la motivación vocacional destaca en primer lugar en Psicología con un $60 \%$ seguida del futuro profesional con un $32 \%$. En cambio, en Económicas estos factores invierten el orden, ocupando el primer lugar el futuro profesional con $49 \%$ seguido de la motivación vocacional con $45 \%$ (Gráfica 5).

\section{MOTIVOS DE ELECCIÓN EN ECONÓMICAS Y PSICOLOGÍA}

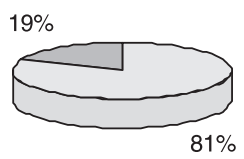

$\square$ Motivos endógenos $\square$ Motivos exógenos

GRÁFICA 4. 
El hecho de obtener una nota insuficiente para realizar la carrera deseada es el factor exógeno que más se elige en Psicología (90\%) y en Económicas, aunque en ésta última, el porcentaje es inferior $(68 \%)$. Además, en Económicas la presión familiar influye también en la elección de carrera (21\%) (Gráfica 0).

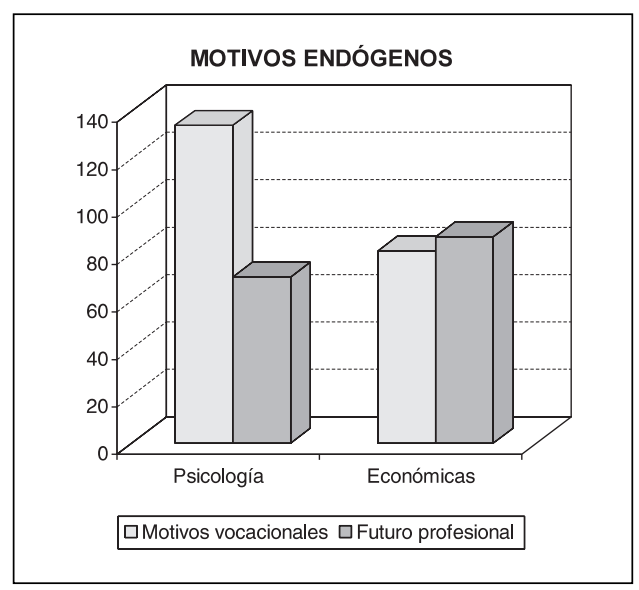

GRÁFICA 5*.

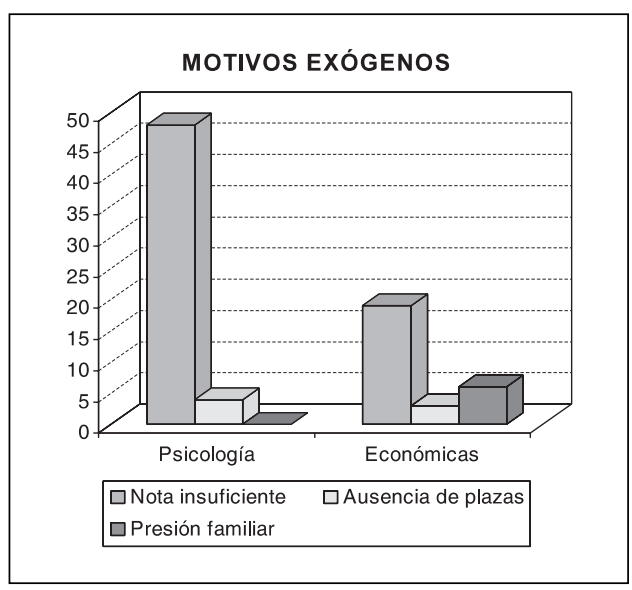

GRÁFICA 6*.

En cuanto a las expectativas que tienen los estudiantes sobre su carrera, se observan diferencias entre ambas licenciaturas: en Psicología se da más importancia a una buena formación, seguida de la salida profesional, de ampliar conocimientos y de obtener un título. En Económicas el factor que prima es la salida profesional, seguida de una buena formación, de la obtención de un título y de la ampliación de conocimientos.

Una vez terminada la carrera, las aspiraciones de los alumnos de ambas Facultades se configuran en el siguiente orden: desempeñar un puesto de trabajo acorde con sus estudios (39\% en Psicología y 50\% en Económicas), cursos de postgrado (32\% en Psicología y 18\% en Económicas), prepararse una oposición (11\% en Psicología y 10\% en Económicas), realizar otra carrera (7\% en Psicología y 9\% en Económicas) y ocupar cualquier puesto de trabajo (5\% en Psicología y $8 \%$ en Económicas).

Si comparamos el porcentaje de alumnos que suspenden en junio o en junio y septiembre con aquellos que no suspenden nunca, podemos observar una gran diferencia: sólo un $4 \%$ en Económicas y un 6\% en Psicología aprueban el curso completo en junio, mientras que los alumnos que suspenden se distribuyen de forma más o menos equitativa entre junio y septiembre (Gráfica 7).

El hecho de suspender supone para algunos alumnos un estímulo para seguir estudiando y esforzándose más (en Psicología un $46 \%$ y en Económicas un 26\%) mientras que para otros es motivo de estancamiento ( $12 \%$ en Psicología y $22 \%$ en Económicas), de indiferencia (14\% en Psicología y $8 \%$ en Económicas), provoca mal clima familiar (7\% en Psico-

\footnotetext{
* Estas gráficas están elaboradas a partir del número de sujetos, aunque en el texto se hace referencia al tanto por ciento para una mejor comprensión del lector.
} 


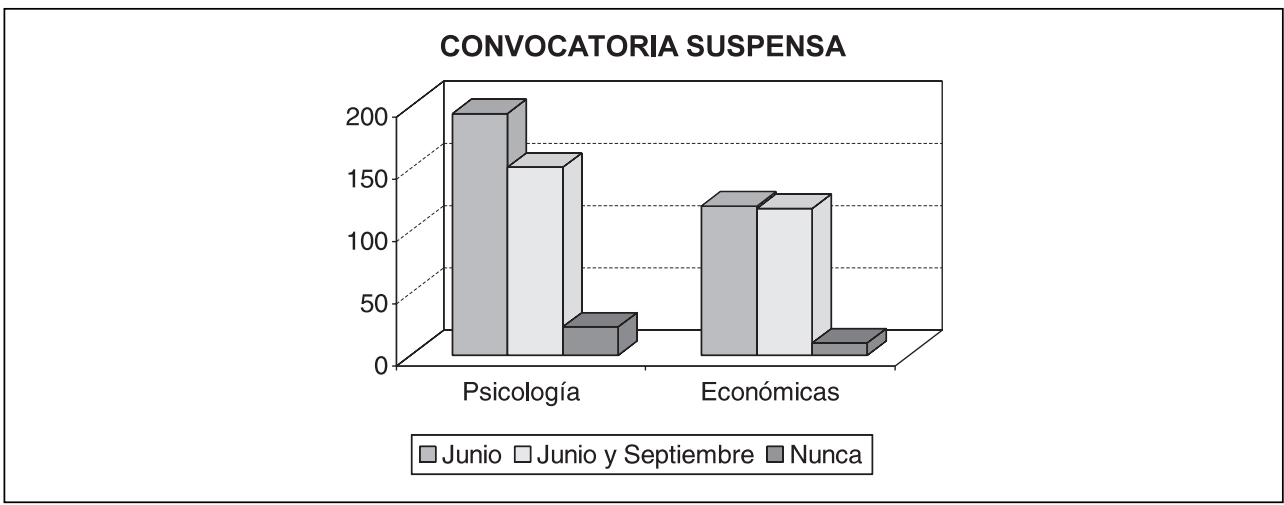

GRÁFICA 7*.

logía y $13 \%$ en Económicas) o lleva al alumno a pensar en abandonar (6\% en Psicología y $13 \%$ en Económicas) (Gráfica 8).

\section{EFECTOS DEL SUSPENSO}

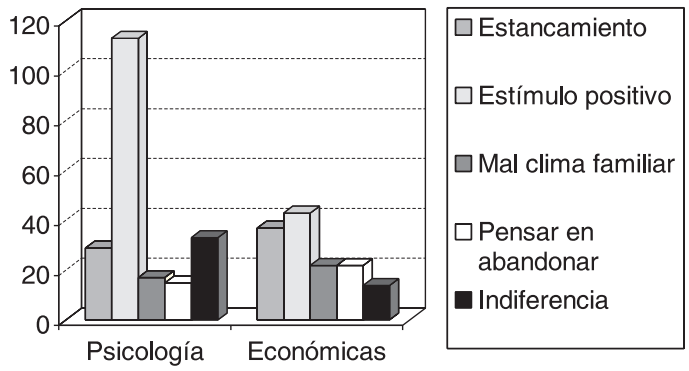

GRÁFICA 8*.

Si analizamos la atribución que los estudiantes realizan sobre las causas que les llevan a suspender, podemos observar la siguiente distribución: en Psicología, se hace una atribución interna, es decir, se señala como causa principal la falta de esfuerzo dedicada al estudio $(30 \%)$. A continuación se distinguen otras, tales como malos profesores $(16 \%)$, desinterés de los alumnos (14\%), dificultad de la materia (10\%) y mala suerte $(8 \%)$. En Económicas, sin embargo, se realiza una atribución tanto interna como externa (un 18\% destaca su falta de esfuerzo y un $19 \%$ la incompetencia del profesor). Además, se consideran la mala suerte (15\%), el desinterés (11\%), la dificultad en la materia (11\%) y la ansiedad en la situación de exámen (10\%) (Gráfica 9).

* Estas gráficas están elaboradas a partir del número de sujetos, aunque en el texto se hace referencia al tanto por ciento para una mejor comprensión del lector. 


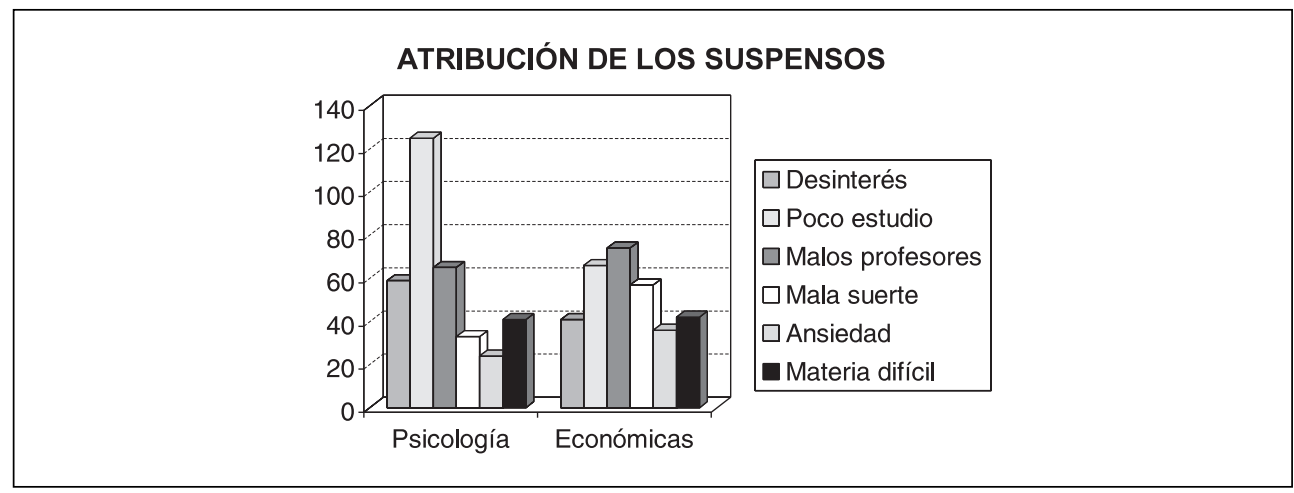

\section{GRÁFICA $9 *$.}

Un aspecto importante que va a influir en el fracaso es la percepción que el alumno tiene de sí mismo como estudiante. En la mayoría de los casos estudiados encontramos una autoimagen positiva: en Psicología, el $61 \%$ se considera un estudiante normal y el $34 \%$ bueno; en Económicas, los porcentajes son $67 \%$ y $28 \%$ respectivamente. A su vez consideran que las características del buen estudiante son las siguientes: comprensión, asimilación, estudiar al día, manejo de fuentes y asistencia a clase (Gráficas 10 y 11).

CARACTERÍSTICAS DEL BUEN ESTUDIANTE

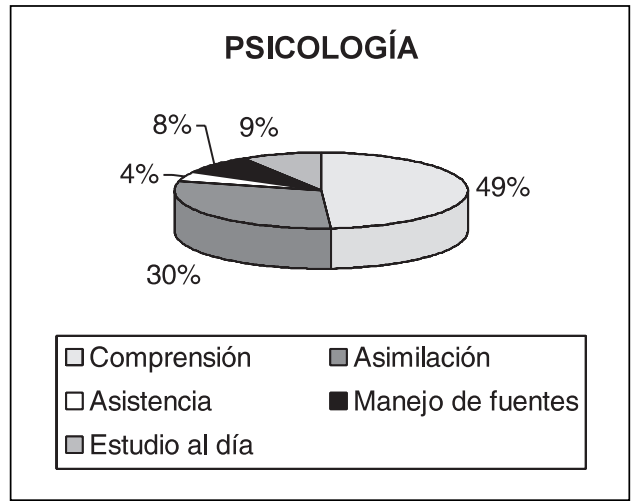

GRÁFICA 10.

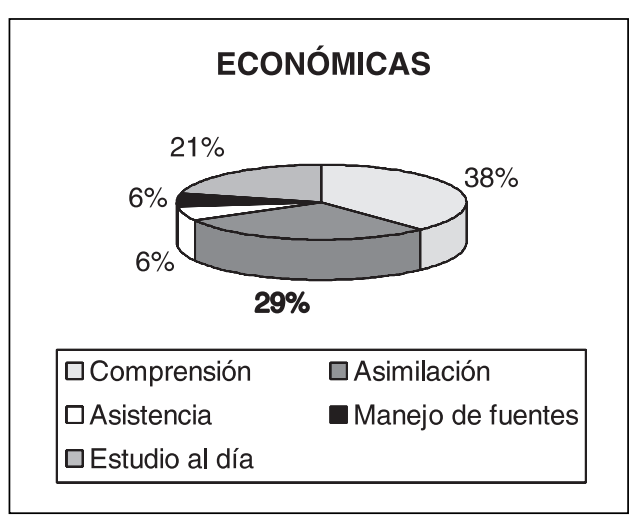

GRÁFICA 11.

En función de la dificultad de la materia, los alumnos comienzan a estudiar con mayor o menor antelación al examen. Así, en Psicología las asignaturas fáciles y de dificultad intermedia se empiezan a estudiar con una semana de antelación, mientras que las difíciles se estudian 15 días antes. Sin embargo, en Económicas, las asignaturas fáciles se estudian una

\footnotetext{
* Estas gráficas están elaboradas a partir del número de sujetos, aunque en el texto se hace referencia al tanto por ciento para una mejor comprensión del lector.
} 
semana antes de la evaluación, las de dificultad media durante 15 días y las difíciles con un mes de anticipación. Son datos que obligan a plantearse con qué ritmo de sosiego y asimilación se realiza el trabajo intelectual en los estudios universitarios de estas Facultades.

Estos estudiantes consideran que el profesor ideal debe cumplir las siguientes características: ser didáctico, motivador, que permita la participación y que sea crítico (Gráfica 12).

\section{CARACTERÍSTICAS DEL PROFESOR}

IDEAL

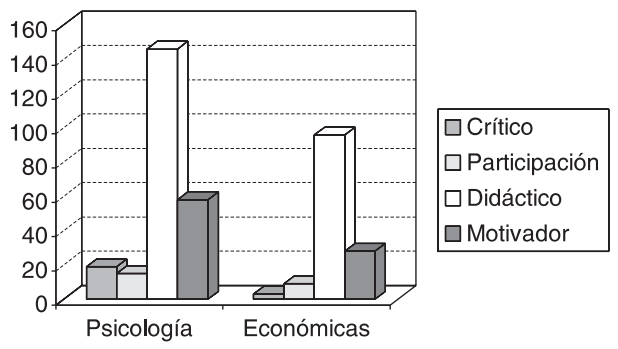

GRÁFICA 12*.

Para concluir este apartado sería interesante destacar que el $47 \%$ de los alumnos que estudian Psicología ha recibido orientación a lo largo de su vida escolar frente a un $63 \%$ de los que cursan Económicas. Entre los estudiantes que no la han recibido, un 83\% en Psicología y un $81 \%$ en Económica hubiera deseado haber sido orientado, deseo que en el caso del Servicio de Orientación de la Facultad de Psicología ha quedado satisfecho mediante solicitud de apoyo para madurar la decisión tomada previamente al inicio de la carrera. En algunos casos ha sido útil para replantearse la decisión tomada, ya sea mediante una afirmación de la misma o, como ha sucedido en algunos casos, para optar por otra abandonando los estudios iniciados. Esta realidad nos obligó a llevar a cabo durante los años de dirección del Servicio de Orientación ciclos de conferencias para que el posible error cometido al «elegir carrera» no se repitiera al tener que «elegir especialidad» antes de iniciar el II Ciclo universitario.

En Psicología, un 76\% cursa la carrera deseada y en Económicas un 64\%.

Prácticamente la totalidad de los alumnos considera necesaria la existencia de un Servicio de orientación en la Universidad (97\% en Psicología y 85\% en Económicas).

\section{Conclusiones}

Tras haber realizado un análisis de los resultados de ambas investigaciones consideramos conveniente hacer mención de las semejanzas y diferencias encontradas.

\footnotetext{
* Estas gráficas están elaboradas a partir del número de sujetos, aunque en el texto se hace referencia al tanto por ciento para una mejor comprensión del lector.
} 
En lo que a la primera investigación se refiere, los motivos endógenos son determinantes a la hora de elegir carrera, siendo la vocación profesional el más destacado, excepto en Ciencias de la Educación. Esto puede deberse a que los alumnos que no conseguían en esos momentos la nota suficiente para cursar la carrera deseada recurren a estos estudios por la escasa nota de corte.

Una vez terminada la carrera, los estudiantes aspiran a ejercer en un puesto de trabajo relacionado con los estudios que han realizado. Este es un aspecto que cabría esperar pero llama la atención el hecho de que elijan la carrera centrándose en su vocación (motivo endógeno) y no tengan tan en cuenta las salidas profesionales (motivo exógeno) que ésta proporciona.

A nivel general, es difícil encontrar alumnos que terminen la carrera sin haber suspendido ninguna asignatura. Sin embargo, en Cc. de la Educación esto no es tan complicado. Una de las razones que podría explicar este hecho es que, comparada con el resto de estudios, Cc. de la Educación es una carrera de menor dificultad. Por otro lado, dicha carrera es la que tiene mayor porcentaje de alumnos que suspenden el curso completo, lo que obliga a plantearse y profundizar en verificar la hipótesis si puede tratarse de alumnos que no la eligieron por vocación sino por falta de nota para estudiar la deseada.

Cuando se produce el fracaso académico, los alumnos de Psicología, Químicas y Farmacia lo atribuyen al poco tiempo dedicado al estudio, a pesar de ser las carreras en las asignaturas comienzan a estudiarse con mayor antelación. Aunque en un principio este resultado pueda parecer contradictorio, si atendemos a la perspectiva subjetiva de los alumnos del tiempo necesario de estudio, podemos ver que no existe tal contradicción ya que, consideran que el tiempo dedicado no es el suficiente. Por otro lado, en Cc. de la Educación atribuyen el fracaso a los malos profesores aunque estudian con menor antelación que sus compañeros de otras facultades. Esta cuestión puede explicarse porque al ser una carrera de menor dificultad, el tiempo requerido para estudiar cada asignatura es también menor y es considerado suficiente por los alumnos. Por ello, el fracaso no puede ser atribuido (por parte de los estudiantes) a la falta de estudio, sino a otros factores.

Los sujetos tienen una autoimagen positiva como estudiantes y, en general, están cursando la carrera deseada. Estos dos aspectos se encuentran relacionados debido a que el estar estudiando lo que les gusta favorecerá el esfuerzo dedicado a las asignaturas, teniendo una mayor probabilidad de éxito y reforzando, a su vez, la autoimagen del alumno.

Por otro lado, la autoimagen del estudiante también estaría en relación con el hecho de aprobar o suspender cada una de las materias estudiadas. Teniendo en cuenta todo esto, creemos que puede afirmarse que aprobar las asignaturas en las que el estudiante está matriculado fomentaría una autoimagen positiva de dicho alumno, mientras que suspender provocaría una autoimagen negativa. Pues bien, esto que sería lo esperado, no es lo que ocurre en los alumnos estudiados sino todo lo contrario: el hecho de suspender les está creando una autoimagen positiva y encima les sirve de estímulo para seguir estudiando.

Centrándonos en la segunda investigación (realizada con los alumnos de Psicología y Económicas durante el curso académico 1999-2000) puede observarse que los principales motivos que guían a los estudiantes en la elección de carrera son endógenos, al igual que sucedía en la muestra estudiada en 1995. De la misma manera, los alumnos de Psicología siguen dando prioridad a la vocación profesional frente a los de Económicas que conceden mayor importancia al futuro profesional que a la vocación, aunque en realidad ambos facto- 
res están muy igualados. Entre los factores exógenos que más influyen podemos destacar la obtención de una nota insuficiente para matricularse en los estudios deseados.

La mayor parte de los estudiantes está realizando la carrera que deseaba. Esto debería favorecer que los alumnos aprobaran en junio, sin embargo, tan solo lo consigue una ínfima proporción. Ello puede atribuirse a que en el rendimiento académico también influyen otros factores como el ambiente de estudio, el ambiente universitario, el clima familiar, la dificultad de cada materia, el tipo de examen, el estilo de enseñanza del profesor, ...

Como observamos en la investigación de 1995, los alumnos siguen teniendo como preferencia desempañar un puesto de trabajo acorde con sus estudios apenas terminan la licenciatura universitaria. Aunque es sabido que las ofertas laborales en Psicología van en aumento, sobre todo en determinadas especialidades como Psicología del Trabajo, hay que tener en cuenta que en Económicas las salidas profesionales no son tan abundantes. A pesar de ello, en este último caso destaca el hecho de que elijan esta carrera guiándose por su vocación.

La atribución de fracaso que realizan los estudiantes de Psicología, sigue siendo interna: la falta de esfuerzo dedicada al estudio. Por su parte, en Económicas se realiza una doble atribución, interna (falta de esfuerzo) y externa (incompetencia del profesorado). A pesar de que en ambos casos consideran que no se esfuerzan lo suficiente y que además sigue existiendo un alto porcentaje de suspensos, los alumnos tienen una autoimagen positiva como estudiantes, manteniendo la línea que quedó marcada en la investigación de 1995. Será interesante poder repetir estos trabajos al cumplirse otros 5 años (curso académico 2004-2005)

El tiempo que los alumnos dedican al estudio varía de una carrera a otra. En Económicas, la tónica predominante en la primera investigación se sigue manteniendo: las asignaturas difíciles se estudian con un mes de antelación, a las de dificultad media se les dedican 15 días y a las fáciles una semana. Sin embargo, en Psicología esto ha cambiado: mientras que en 1995 se seguía la distribución que acabamos de mencionar, actualmente los alumnos dedican a las asignaturas fáciles o de dificultad intermedia una semana y a las difíciles 15 días. Este aspecto llama la atención (en Psicología) de forma negativa porque hoy día, aún obteniendo en la propia formación mayores conocimientos sobre cómo aprovechar al máximo el tiempo dedicado al estudio, estos conocimientos teóricos no se ponen en práctica. En el resto de carreras estudiadas no podemos mencionar este aspecto, ya que, en la segunda investigación no se mantuvieron las carreras de partida y, por tanto, no podemos comprobar si en ellas se ha producido alguna variación.

Algo que cada vez está cobrando mayor importancia es el hecho de recibir Orientación a lo largo de la vida académica. Sólo aproximadamente la mitad de los alumnos estudiados han sido orientados, sin embargo, prácticamente la totalidad de ellos destacan que les hubiera gustado haberla recibido y además, consideran esencial la existencia de un Servicio de Orientación que lleve a la realidad lo que en los Departamentos Universitarios del área de Orientación se postula como elemento central del trabajo del profesorado y de la capacitación de los alumnos de Facultades como las de Educación y Psicología que han de proporcionar a la sociedad los futuros Orientadores. Sería lamentable tener que reconocer el viejo dicho del cuchillo de palo en casa del herrero.

A pesar de todo, esta investigación podría haber aportado más datos y mayores resultados si los grupos elegidos en los períodos 1994-1995 y 1999-2000 hubieran pertenecido a las mismas carreras ya que con las muestras utilizadas sólo se ha podido ver la evolución 
del fracaso en la carrera de Psicología. La facilidad de tener a estos alumnos cerca ha sido un factor determinante. En otras Facultades la propuesta no tuvo la suficiente resonancia para ser llevada a cabo. Por otra parte, consideramos que sería conveniente realizar una comparación entre las diferentes especialidades dentro de cada carrera en futuros estudios o investigaciones.

\section{Una última reflexión}

El fenómeno del fracaso escolar, tanto en la Universidad como en otros niveles de la enseñanza, es un cajón de sastre donde casi todo es posible. Desde el «bache» en los estudios hasta el "percance del suspenso», sin olvidar el propio fracaso que, espigando en los distintos autores que se han acercado a él, puede llevar a abandono del camino emprendido, se usa con excesiva ligereza y, tal vez, con efectos muy destructivos, lo que hay que entender por tal. Nosotros hemos avanzado nuestra idea (Ríos, 1972) y parece que, aún al cabo de tanto tiempo, mantiene su vigencia. Esto, evidentemente, no alivia la situación sino que la agrava. Porque habría que dar un paso más. ¿En qué sentido? Sencillamente en el de ampliar el concepto para dar entrada a «algo» que se olvida. En la legislación educativa española estuvo presente un concepto que no se ha tenido en cuenta con la suficiente amplitud: se trata de distinguir entre rendimiento «suficiente» y «satisfactorio». Ni padres ni educadores se alarman cuando el rendimiento del hijo o el alumno le permite continuar su proceso de aprendizaje sin especiales sobresaltos ni serias amenazas inmediatas. Se aprueba, aunque a veces sea con trancas y barrancas, y parece que no ha sucedido nada. En tales casos se está hablando de un «rendimiento suficiente». ¿Suficiente para qué...? Para continuar, para no alarmarse en exceso, para no plantearse la disyuntiva de abandonar o no abandonar los estudios iniciados. Pero con tal enfoque se oculta una realidad tremendamente amenazante: hay muchos alumnos -tal vez demasiados, pero que nunca se hacen objeto de estudio e investigaciones- que «rinden» lo suficiente aunque de manera «insatisfactoria». Con otras palabras: podían y debían dar mejores resultados que los que obtienen. Coloquialmente podemos decir que hay estudiantes -también en la Universidad- que tienen capacidad y dotación para rendir «sobresalientemente» o «notablemente» y se quedan al nivel de rendir con lo mínimo que se encarna en la calificación de «aprobado». Quien no da todo lo que puede, también fracasa. Aunque estos «fracasos» pasan desapercibidos para los propios alumnos, para los padres y para lo responsables de la enseñanza.

Creemos que hay que plantearse abierta y claramente este aspecto. Pero este punto de vista constituye un nuevo desafío. La «realidad» del fracaso en la Universidad tiene mucho que ver con esta vertiente que acabamos de apuntar.

El exceso de capacidad intelectual que se pierde es un verdadero fracaso. Pero esa realidad no se evalúa ni se tiene en cuenta. Las estadísticas, por supuesto, ignoran esa franja de la población que debiera tenerse muy en cuenta. Esperamos y deseamos que alguien se atreve a indagar lo que se oculta tras los rendimientos insatisfactorios, aunque se camuflen tras el ropaje del rendimiento suficiente.

$$
* * *
$$

Al comparar los resultados con otras investigaciones, en especial la llevada a cabo por la profesora Latiesa (1992), hemos observado que a pesar de que ambas tratan el fracaso 
universitario como tema común, la mayoría de los factores que se emplean para estudiarlo son diferentes. Esto apoya la idea de que no existe una definición completa y unánime de dicho concepto. Por tanto, cabría señalar la conveniencia de seguir estudiando e investigando en este campo. Sólo así podrá lograrse un mayor número de datos que hagan posible conseguir un pleno consenso y llegar algún día a conocer cuáles son realmente las principales causas del fracaso para abordarlas y, por qué no, acabar con él.

\section{Referencias bibliográficas}

Avanzini, G. (1979): El fracaso escolar. Herder, Barcelona.

Dorsch, F. (1994): Diccionario de Psicología. Herder. Barcelona.

García Hoz, V. (1974): Diccionario de Pedagogía. Tomo II. Labor. Barcelona.

González Tirados, R. M. (1989): Análisis de las causas del fracaso escolar en la U.P.M. Madrid: Centro de publicaciones.

Latiesa, M. (1992): La deserción universitaria. Siglo XXI de España Editores, Madrid.

Martínez, B. (1983): Causas del fracaso escolar y técnicas para afrontarlo. Narcea, Madrid.

Martínez, B. (1988): La familia ante el fracaso escolar. Narcea, Madrid.

Martínez-Otero, V. (1997): Los adolescentes ante el estudio. Fundamentos, Madrid

Mc Clelland, D. C. (1989): Estudio de la motivación humana. Narcea, Madrid

Portellano Pérez, J. A. (1989): Fracaso escolar: diagnóstico e intervención, una perspectiva neuropsicológica. CEPE. Madrid, Ciencias de la educación preescolar y especial.

Ríos, J. A., Perearnau, M. A. (1972): Fracaso escolar y vida familiar. Marsiega, Madrid.

Rubio Jerónimo, A., Ferre Rubio, F. y Rojo Calvo, C. (1989): «El fracaso escolar en B.U.P. y C.O.U.: un análisis cuantitativo». Educadores.

Rubio Jeronimo, A., Rojo Calvo, C. y Ferre Rubio, F. (1989): «El fracaso escolar en B.U.P. y C.O.U.: un estudio empírico de la atribución causal». Educadores.

Tierno Jiménez, B. (1997): Del fracaso al éxito escolar. Plaza y Janés, Barcelona.

Wall, W. D., Schonell, F. J., Willard, C. O. (1970): El fracaso escolar. Paidós, Buenos Aires.

Weinstein, G. W., Yelon, S. L. (1988): La psicología en el aula. Trillas, Méjico.

Fecha de recepción: 15-01-03

Fecha de revisión: 10-09-03

Fecha de aceptación: 27-10-03 